\title{
Falha de Serviços: Mapeamento de 10 Anos de Produção Científica
}

\section{Service Failure: Mapping for 10 Years of Scientific Production}

\author{
Marcelo Carvalho \\ Universidade Nove de Julho -UNINOVE- Brasil \\ marcelocarvalho.gestor@gmail.com \\ ORCID: 0000-0003-2121-4318 \\ Evandro Luiz Lopes \\ Universidade Nove de Julho -UNINOVE- Brasil \\ elldijo@uol.com.br \\ ORCID: 0000-0002-2780-4215 \\ Otávio Bandeira de Lamônica Freire \\ Universidade de São Paulo - EACH/USP \\ otfreire@usp.br \\ ORCID: 0000-0002-9008-4273 \\ Cristiane Drebes Pedron \\ Universidade Nove de Julho -UNINOVE- Brasil \\ cdpedron@gmail.com \\ ORCID: 0000-0002-9920-3830
}

Submetido em 25/11/2018; Aprovado em 12/03/2019

\begin{abstract}
Resumo
Objetivo - 0 objetivo deste artigo é mapear o arcabouço intelectual da pesquisa sobre falha de serviços por meio do levantamento dos estudos publicados sobre a temática entre 2008 e 2017. Metodologia Estudo bibliométrico, contendo análises de citação, cocitação e pareamento bibliográfico. Principais resultados - Os resultados indicam que o tema permanece atual, relevante, com aumento de publicações nos últimos anos. As três principais abordagens observadas na trajetória de estudos sobre o tema são: qualidade do encontro, percepção de sentimentos, e encontros de recuperação. Observou-se ainda que a partir de 2008 houve uma expansão nas vertentes utilizadas nas abordagens, sendo: recuperação de clientes, resposta emocional, contabilidade mental, capacidade de controle, lealdade, resposta à recuperação, e justiça percebida. Contribuições acadêmicas -Oferece um mapeamento sobre a trajetória dos estudos realizados. A análise da amostra possibilita indicar que, se por um lado se construiu uma robusta literatura sobre a ocorrência das falhas de serviço (cocitação), o estudo sobre a recuperação pós falhas ainda possui lacunas (pareamento). 0 não esgotamento do tema decorre do fato de consumidores responderem de modo diferente, frente aos diferentes tipos de recuperação. Contribuições práticas Este artigo oferece aos gestores um guia dos principais artigos sobre falha de serviços, bem como um alerta para que considerem esforços contínuos na reparação de inevitáveis falhas de serviço.
\end{abstract}

Palavras-Chave: Falha de serviços; bibliometria; recuperação de clientes.

\begin{abstract}
Objective - The aim of this article is to map the intellectual framework of the research on service failure by means of a survey of the studies published on the theme between 2008 and 2017. Methodology Bibliometric study, containing analysis of citation, cocitation and bibliographic coupling. Main results The results indicate that the theme remains current, relevant, with an increase in publications in recent years. The three main approaches observed in the trajectory of studies on the theme are: quality of the meeting, perception of feelings, and recovery meetings. It was also observed that since 2008 there has been an expansion in the approaches used, such as: customer recovery, emotional response, mental accounting, control capacity, loyalty, response to recovery, and perceived justice. Academic contributions - Provides a mapping of the trajectory of the studies carried out. The analysis of the sample makes it possible to indicate that, if, on the one hand, a robust literature was built on the occurrence of service
\end{abstract}


failures (cocitation), the study on post-failure recovery still has gaps (matching). The non-exhaustion of the theme stems from the fact that consumers respond differently to different types of recovery. Practical contributions - This paper gives managers a guide to the main papers on service failure, as well as an alert to consider ongoing efforts to repair unavoidable service failures.

Keywords: Service failure; bibliometrics; customer recovery

\section{Introdução}

Consumidores contemporâneos mantêm uma postura de destaque nas relações comerciais, forçando as empresas a tornarem-se mais atrativas, de modo a propiciar boas experiências de compra para seus clientes. Embora as ações utilizadas no ambiente comercial visem atrair e reter clientes, existe uma série de fatores que podem gerar uma experiência negativa de compra. A falha de serviços é um desses exemplos, e pode ser definida como um serviço que não atendeu às expectativas dos clientes (Agarwal, Mehrotra, \& Barger, 2016). Mesmo que empresas tenham a precaução com a melhora na qualidade de seus produtos e serviços, estudos apontam que falhas ocorrerão, e irão afetar o nível de satisfação dos consumidores (Tarofder, Nikhashemi, Azam, \& Haque, 2016).

Clientes podem exprimir diferentes reações ao vivenciar falhas de serviço, tais como mudanças no nível de satisfação, reações emotivas (como raiva, descontentamento ou remorso) e comportamentais (como reclamação, boca-a-boca negativa, ou mudar de fornecedor) (Huang, Hung, Fu, Hsu \& Chiu, 2015). Esforços para a recuperação do consumidor, de modo a reduzir os impactos causados pelas falhas de serviço, devem estar entre as prioridades das empresas que queiram manter uma relação duradoura com seus clientes.

A busca por relacionamentos mais duradouros com os consumidores parece ser um consenso entre as empresas. $O$ alto custo na aquisição de novos clientes faz com que a retenção da carteira de clientes atual ganhe destaque (Hennig-Thurau, Gwinner, \& Gremler, 2002). Diante da inevitável ocorrência de falhas de serviço (Tarofder et al., 2016), é necessário que as empresas possam oferecer respostas assertivas aos consumidores, de modo a realizar uma recuperação pós falha mais efetiva.

Nesse contexto, o presente estudo tem por objetivo mapear o arcabouço intelectual da pesquisa sobre falha de serviços por meio do levantamento dos estudos publicados sobre a temática entre 2008 e 2017, e assim identificar as principais vertentes utilizadas como abordagem. Este trabalho visa aprofundar o entendimento sobre os efeitos produzidos por falhas de serviço, bem como apontar avenidas de pesquisas futuras. Diante da proposição de que as falhas são intrínsecas aos serviços, parece ser oportuno que as empresas, não só entendam as causas das falhas, mas também identifiquem as falhas mais importantes para a qualidade do serviço ofertado.

Assim, em função da relevância do tema, foi realizada uma pesquisa bibliométrica, como forma de medir os índices de produção e divulgação do conhecimento científico (Silva, Santos, Brandão, \& Vils, 2016; Araújo, 2006), de modo a mapear os estudos desenvolvidos sobre falha de serviços nos últimos 10 anos. Para o desenvolvimento deste estudo, foram selecionados 199 artigos no portal Web of Science. Foram realizadas análises de citação, indicando a frequência de citações utilizadas na pesquisa acadêmica e assim o grau de importância do trabalho, de cocitação, que, por meio da citação conjunta, indica as conexões entre os trabalhos, e indica uma estrutura intelectual, e de pareamento bibliográfico, que identifica citações compartilhadas por, ao menos, dois documentos da amostra. Este trabalho possui cinco seções, além da introdução, sendo (2) referencial teórico sobre falhas de serviço e satisfação; (3) metodologia utilizada nesta pesquisa; (4) análise dos resultados obtidos; e, na quinta seção, são apresentadas as considerações finais, limitações e sugestões para pesquisas futuras.

\section{Referencial teórico}

Neste capítulo são expostos os aportes teóricos que embasam esta pesquisa. 0 primeiro tópico trata da falha de serviços, em seguida aborda-se a satisfação do consumidor.

\subsection{Falhas de Serviço}

Falha de serviços pode ser definida como aspectos da entrega do serviço que não atenderam às expectativas dos consumidores (Agarwal et al., 2016). A atividade de prestação de serviços apresenta três características críticas para a percepção de qualidade do cliente: intangibilidade, depende da percepção subjetiva do cliente; participação do cliente no processo, diminuindo o controle do prestador 
de serviços; e produção e consumo simultâneos, impossibilita o controle antecipado do serviço (Parasuraman, Zeithaml, \& Berry, 1990).

Visando compreender a falha percebida, consumidores utilizam qualquer informação disponível para justificar o ocorrido (McColl-Kennedy \& Sparks, 2003). Na ausência de informações reais sobre a causa da falha, podem atribuir uma avaliação pior do que o ocorrido de fato (Bies \& Shapiro, 1987). Consumidores atribuem responsabilidade por falhas com base em seus traços de personalidade dominantes (Agarwal et al., 2016). Assim, se os clientes possuem uma natureza intolerante, eles tendem a sentir que um provedor de serviços poderia ter resolvido o problema ou evitado a falha, mas não conseguiu fazê-lo (Agarwal et al., 2016). Após constatar uma falha de serviço, um consumidor leal tende a gerar uma punição para a empresa envolvida, mesmo que não tenha ganhos materiais (Grégoire \& Fisher, 2008).

Empresas devem buscar a reparação da falha no serviço e, assim, recuperar consumidores insatisfeitos, prevenindo que os clientes abandonem a empresa (Orsingher, Valentini, \& de Angelis, 2010). Nesse sentido, a interação com os clientes nos momentos após a falha do serviço tem um papel determinante para o sucesso da recuperação da falha (Mattila \& Patterson, 2004). A recuperação de uma falha oferece uma segunda chance para os provedores de serviços (Nwokorie, 2016). Esses clientes podem realizar um boca a boca positivo para a empresa (Choi \& Choi, 2014). Quando uma empresa demonstra empatia e sinceridade ao abordar uma falha no serviço, os problemas tendem a ser mitigados (Agarwal et al., 2016).

No entanto, estudos indicam que clientes com forte relacionamento com a empresa, e que não recebam recuperações após suas queixas on-line, sentem-se mais traídos, e seu desejo de vingança mantem-se presente ao longo do tempo (Grégoire, Tripp \& Legoux, 2009). A gravidade da falha cometida também influencia a perda percebida pelo consumidor (Nikbin, Marimuthu, Hyun, \& Ismail, 2015). A gravidade da falha pode influenciar negativamente as emoções dos consumidores e aumentar o esforço na tentativa de recuperação da satisfação do consumidor (Lorenz, Johnson, \& Barakat, 2017).

\subsection{Satisfação}

A teoria da desconfirmação de Oliver (1980) é o alicerce para a literatura sobre a satisfação do consumidor. Para ele, o nível de satisfação resulta das diferenças existentes entre desempenho real e esperado, de um produto ou serviço. A satisfação é melhor compreendida se vista como um processo, na qual as percepções do consumidor devem ser consideradas antes, durante e depois do consumo (Oliver \& Swan, 1989). A satisfação advém da comparação feita pelos consumidores entre suas expectativas e suas percepções de desempenho do produto (Oliver, 1980). Portanto, satisfação do cliente é o retorno dado pelo consumidor ao atendimento recebido, baseado em suas expectativas (Zeithaml \& Bitner, 2003). Se os clientes avaliarem que receberam serviços que atenderam às suas expectativas, eles terão opiniões e emoções positivas sobre esses serviços (Park, 2019).

O conceito denominado de "momento da verdade" está associado a interação do consumidor com a experiência de serviço, e é uma oportunidade para o prestador do serviço tentar influenciar de forma positiva a formação da satisfação do cliente (Lovelock \& Wirtz, 2007). Se os problemas forem antecipados, a empresa pode fornecer explicações com antecedência, de tal forma que os clientes estejam preparados para a possível falha e entendam por que o problema ocorreu (Agarwal et al., 2016). A satisfação é um fator importante para o desenvolvimento da lealdade do consumidor (Hung, Cheng, \& Chiu, 2018).

Por outro lado, a maioria dos clientes insatisfeitos simplesmente mudam de fornecedor (Ro, 2014). Em situações mais extremas, clientes que vivenciam de forma negativa suas relações de serviço, podem utilizar a internet para realizarem suas queixas de modo público, trazendo consequências prejudiciais para as empresas (Ward \& Ostrom, 2006).

\section{Método}

0 presente estudo tem uma abordagem descritiva e quantitativa. Foi realizada uma análise bibliométrica, que utiliza conhecimentos estatísticos e matemáticos para medir os índices de produção e divulgação do conhecimento científico (Araújo, 2006). Possibilita um mapeamento da estrutura do campo pesquisado, estabelecendo as conexões existentes entre os artigos publicados, oferecendo assim, uma visão abrangente sobre o tema (Zupic, \& Čater, 2015). Seu uso contribui para a compreensão de novos temas e tendências (Silva et al., 2016), auxiliando o campo a estruturar novas avenidas de teoria e pesquisa. 
Para o desenvolvimento desse estudo, foram realizadas análises de citação, cocitação e pareamento bibliográfico. A análise de citações investiga as relações entre os documentos citantes e citados, indicando autores mais citados, autores mais produtivos e periódicos com maior número de publicações no tema (Araújo, 2006). A análise de cocitação e a análise de pareamento bibliográfico foram largamente utilizadas em estudos bibliométricos nas últimas décadas. Podem ser descritas como relações intertextuais entre publicações científicas que refletem alguma semelhança entre os artigos cocitados ou pareados (Vogel, \& Güttel, 2013). A análise de cocitação é uma medida de similaridade, indicando a periodicidade com que duas referências são citadas em conjunto (Zupic, \& Čater, 2015). A análise de cocitação é uma maneira de articular documentos, registrando a quantidade de trabalhos que citaram algumas publicações em conjunto, de modo que os artigos muito cocitados tendem a se agrupar quando mapeados (Lin \& Cheng, 2010). Quanto maior a ocorrência de cocitações, maior será a similaridade entre as referências (Ramos-Rodriguez \& Ruíz-Navarro, 2004). Os artigos são cocitados quando incluídos em uma mesma lista de referências (Vogel, \& Güttel, 2013). A análise de cocitação não é estática, de modo que as frequências de cocitação podem variar em função do desenvolvimento do estado da arte (Zupic, \& Čater, 2015; Vogel, \& Güttel, 2013).

O pareamento bibliográfico é uma medida de associação entre duas publicações citadas, estabelecida por referências feitas pelos autores dos documentos envolvidos e, portanto, é intrínseca a esses documentos (Vogel, \& Güttel, 2013). O pareamento ocorre quando uma mesma referência está presente em dois artigos distintos (Egghe \& Rousseau, 2002). A intensidade do pareamento aumenta à medida que as referências bibliográficas dos dois documentos se sobrepõem (Vogel, \& Güttel, 2013). A análise de pareamento bibliográfico tem seu foco dirigido para novas vertentes de estudo (Vogel, \& Güttel, 2013).

Assim, a cocitação avalia a trajetória do tema, e o pareamento indica a tendência do que foi publicado (Zupic, \& Čater, 2015). A Figura 1 apresenta a diferença entre cocitação e pareamento bibliográfico.

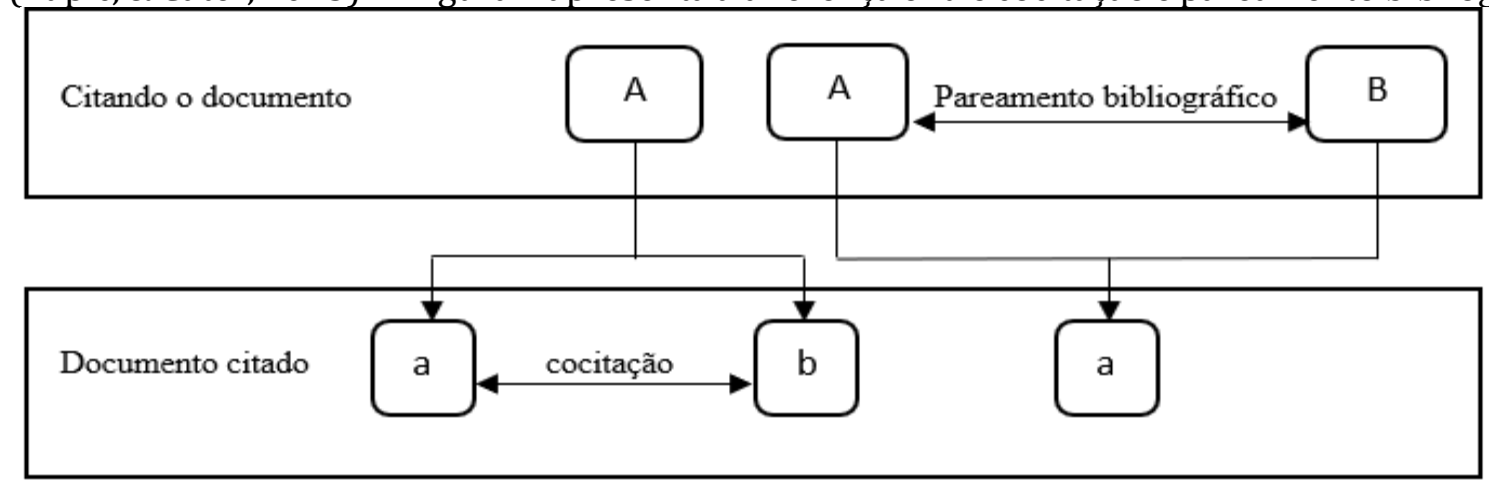

Figura 1: Cocitação e pareamento bibliográfico.

Fonte: Adaptado de Vogel e Güttel (2013).

O ponto de partida para esta pesquisa foi a seleção de artigos que consideraram a falha de serviço e a satisfação no estudo do comportamento do consumidor. A Web of Science foi a base de dados utilizada para a presente pesquisa, pois é uma das mais utilizadas em pesquisas bibliométricas, e por já oferecer informações sobre relevância dos periódicos e número de citações (Silva et al., 2016). Realizou-se um levantamento a partir dos termos "service failure" e satisfaction, resultando em 480 documentos. Decidiu-se então selecionar apenas artigos, reduzindo a lista para 404 artigos.

0 terceiro critério estabeleceu o corte temporal, selecionando apenas artigos publicados nos últimos dez anos (2008-2017), pré-selecionando, 332 artigos. A decisão acerca do recorte temporal de 10 anos está baseada nas recomendações da literatura. Estudos bibliométricos devem ser conduzidos em um recorte temporal próximo, para que os dados analisados sejam um espelho do estado atual do estudo do fenômeno (Katz \& Hicks, 1997), e ao mesmo tempo abrangente (Leydesdorff, 2009) para que o estudo possa indicar o desenvolvimento do campus do conhecimento. Em geral, um recorte temporal entre 10 e 20 anos parece ser adequado para a condução de estudos desta natureza (Andrés, 2009). Em seguida considerou-se apenas as Categorias do Web of Science Business e Management, obtendo assim, um resultado de 244 artigos. Por fim, realizou-se a leitura dos abstracts dos 244 artigos selecionados, de modo a confirmar se todos tratavam de falha de serviços. Por não apresentar conformidade com os cri- 
térios estabelecidos, 45 artigos foram descartados, resultando assim, em uma base de 199 artigos validados.

Foram realizadas três análises para que o objetivo deste estudo fosse alcançado: citação, cocitação, e pareamento bibliográfico. A análise de citação, objetivou determinar, entre 2008 e 2017, os principais autores que publicaram sobre o tema, o volume de publicações, os periódicos que mais publicaram sobre o tema, e os artigos mais citados. A segunda análise realizada foi a de cocitação, que mede a ocorrência em que um par de referências é citado conjuntamente (Vogel, \& Güttel, 2013). Para tal, foi realizada uma análise fatorial exploratória, utilizando uma matriz de cocitação criada no software Bibexcel e conduzida com o software SPSS (versão 18).

Para a análise fatorial, utilizou-se o método de rotação Varimax, de modo a agrupar os artigos que se equipararam, e foram consideradas apenas as cargas fatoriais superiores a 0,50 . Os fatores aceitos foram validados com a leitura dos títulos, abstracts, introdução e discussão dos artigos selecionados. 0 pareamento bibliográfico foi a terceira análise realizada, visando identificar citações compartilhadas por, ao menos, dois documentos da amostra. Os procedimentos realizados foram os mesmos utilizados na análise de cocitação. Para a criação das redes relacionais, foi utilizado o software Ucinet.

\section{Análise dos resultados}

Nesta seção serão apresentados os resultados obtidos neste trabalho. Com o objetivo de mapear os estudos desenvolvidos sobre falha de serviços nos últimos 10 anos, foi realizada uma análise bibliométrica com base em 199 artigos extraídos da base Web of Science.

\subsection{Análise de Citação}

Os 199 artigos da amostra citaram, no total, 6.788 artigos em suas referências. Selecionamos então, dentre as referências utilizadas, apenas os artigos que receberam mais citações. Como ponto de corte para a seleção dos artigos citados, foi estabelecido um limite mínimo de 20 citações. A seleção resultou nos 50 artigos mais citados, e que serão utilizados para as análises.

A primeira análise visou estabelecer o número de publicações em função dos anos abordados na pesquisa, conforme Figura 2. 0 maior número de publicações ocorreu em 2017, com 31 publicações, seguido por 2016, com 30 artigos publicados. A concentração de publicações nos últimos três anos do período selecionado, indica atualidade e relevância crescente do tema.

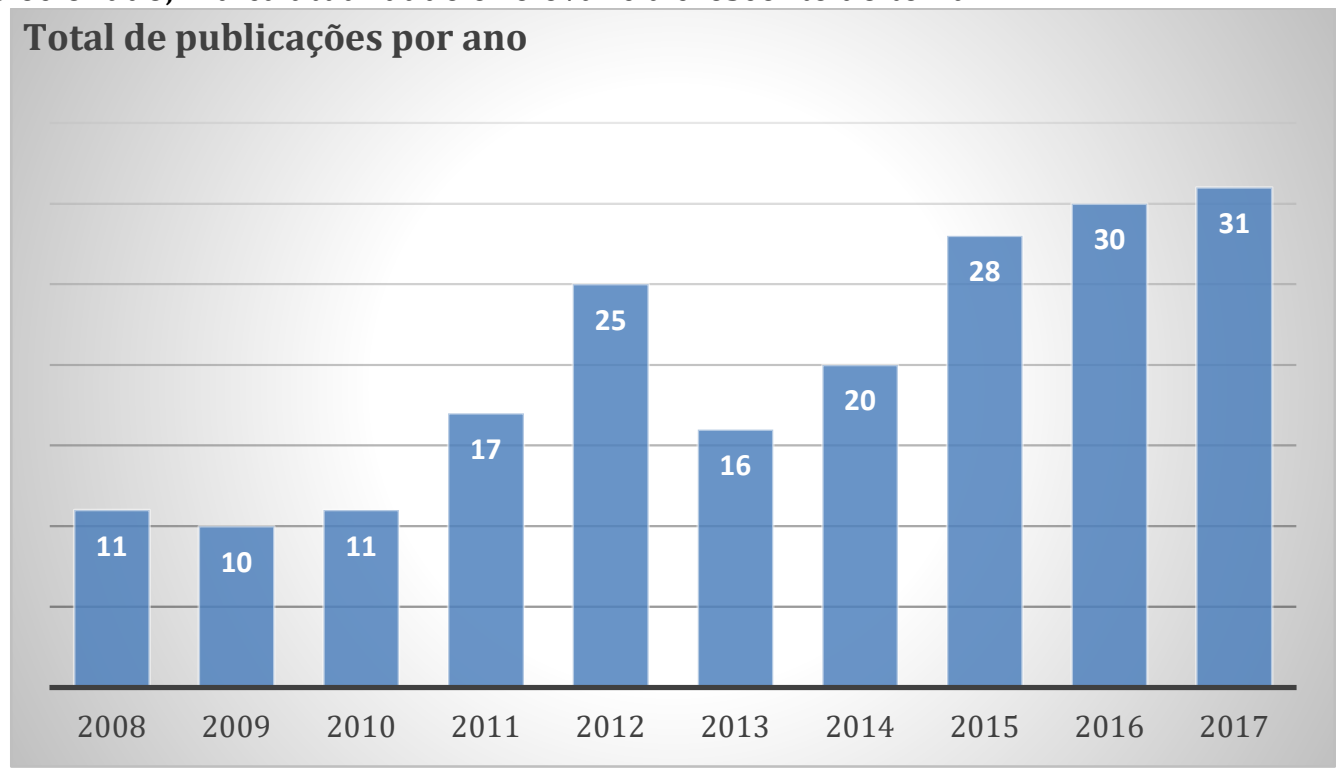

Figura 2: Evolução de publicações.

Fonte: Elaborado pelos autores (2018).

O volume de citações para os artigos selecionados nesta pesquisa, também acompanha essa tendência, atingindo um total de 745 citações no ano de 2017, e, no período de 10 anos, um total de 2.833 citações, como observado na Figura 3. 
Número de citações por ano

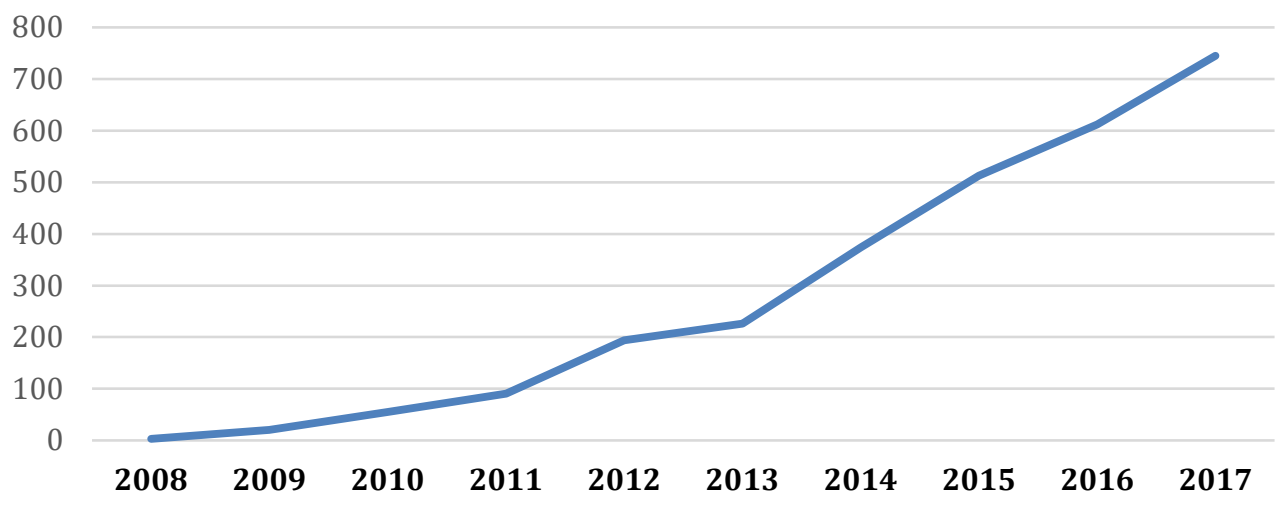

Figura 3: Número de citações por ano.

Fonte: Elaborado pelos autores (2018).

Um ponto a ser observado, no que tange à relevância das publicações, é o periódico em que o artigo foi publicado. Nesse sentido, determinou-se a relação dos Journals que publicaram os artigos selecionados, relacionados em ordem decrescente quanto ao número de publicações, e com indicação de seu fator de impacto em cinco anos. Os 199 artigos publicados no período estão distribuídos em 56 periódicos. A Tabela 1 apresenta os 10 periódicos com maior número de artigos publicados no período estudado. 0 Journal of Services Marketing, fator de impacto 2.817, foi o periódico com maior número de publicações, 30 artigos, e o Journal of the Academy of Marketing Science, é o que possui maior fator de impacto, com fator 9.81. Quatro desses periódicos - Journal of Services Marketing, Journal of Service Research, Service Industries Journal, e Service Business, são específicos da área de serviços. Os demais periódicos são referências mais gerais, tanto em Marketing, quanto em Administração, amplamente citados e consultados.

\section{Tabela 1 - Journals com maior número de publicações}

\begin{tabular}{llc}
\hline Periódicos & Fator de impacto 5 anos & Artigos Validados \\
\hline Journal of Services Marketing & 2.817 & 30 \\
Journal of Business Research & 3.689 & 13 \\
Service Industries Journal & 7.267 & 13 \\
Journal of the Academy of Marketing Science & 9.81 & 12 \\
Journal of Service Research & 7.099 & 11 \\
Service Business & 2.214 & 8 \\
European Journal of Marketing & 2.545 & 7 \\
Journal of Retailing & 5.53 & 7 \\
Journal of Service Management & 5.407 & 6 \\
Managing Service Quality & 3.466 & 5
\end{tabular}

Fonte: Elaborado pelos autores (2018).

Para maior entendimento sobre a estrutura de conhecimento desenvolvida, é importante determinar os autores com maior produtividade sobre o tema no período. Um total de 436 autores participaram da amostra. Os 20 autores com maior número de publicações nos dez anos pesquisados, somam 76 artigos como apresentado na Tabela 2 Grégoire Yany, Ph.D. em Marketing pela University Western Ontario é o autor mais prolífico, com sete artigos publicados. Os seis autores com maior nível de produção, tiveram a maioria de seus trabalhos publicados após 2012, sendo que, Gregóire, e Vasquez-Casielles, Iglesias e Varela-Neira, são os que publicaram em 2017, posicionando-se como expoentes das pesquisas recentes sobre o tema.

Em seu artigo, Gregóire (2017) assevera que empresas que oferecem compensação (reparações tangíveis) ou melhoria de processos organizacionais demostram desempenho mais estável. Por meio de uma pesquisa quantitativa, observou dados de 212 empresas de capital aberto ou subsidiárias, por um 
período de 160 semanas, entre os anos de 2001 a 2013. Vasquez-Casielles, Iglesias e Varela-Neira (2017) apontam a importância da co-criação e da comunicação do processo de recuperação de serviços nos quais os clientes são informados sobre a adoção de soluções para resolver a causa da falha. Eles realizaram um estudo quantitativo com 224 atacadistas da indústria de alimentos e bebidas da Espanha, testando a eficácia das diferentes estruturas de governança para salvaguardar investimentos específicos feitos pelo distribuidor. Esses dados reforçam a tendência crescente de pesquisas sobre o tema.

Tabela 2 - Principais autores.

\begin{tabular}{c|l|c|c|l|c}
\hline RANK & \multicolumn{1}{|c|}{ AUTOR } & $\begin{array}{c}\text { ARTIGOS PUBLI- } \\
\text { CADOS }\end{array}$ & RANK & \multicolumn{1}{|c}{ AUTOR } & PUBLICAÇÕES \\
\hline 1 & Gregoire, Y. & 7 & 11 & Balaji, M.S. & 3 \\
2 & Vazquez-Casielles, R. & 6 & 12 & Boshoff, C. & 3 \\
3 & Varela-Neira, C. & 6 & 13 & Brady, M.K. & 3 \\
4 & Mattila, A.S. & 5 & 14 & Choi, B. & 3 \\
5 & Iglesias, V. & 5 & 15 & Chih, W.H. & 3 \\
6 & De Matos, C.A. & 4 & 16 & Gohary, A.. & 3 \\
7 & Gelbrich, K. & 4 & 17 & Marimon, F. & 3 \\
8 & Patterson, P.G. & 3 & 19 & Mccoll-Kennedy, J.R. & 3 \\
9 & Tsarenko, Y. & 3 & 20 & Alizadeh H. & 2 \\
10 & Van Vaerenbergh, Y. & \multicolumn{3}{c}{} \\
\hline
\end{tabular}

Fonte: Elaborado pelos autores (2018).

No ranking de artigos mais citados apresentados na Tabela 3 , o paper When Customer Love Turns into Last Hate: The Effects of Relationship Strength and Time on Customer Revenge and Avoidance, publicado em 2009, no Journal of Marketing, por Gregóire, Tripp e Legoux, aparece em primeiro lugar com 179 citações. Esse artigo explora os efeitos do tempo e da força de relacionamento na evolução da vingança e evasão dos clientes em contextos de queixa pública online. Os autores concluem que, com o passar do tempo, existe uma diminuição no desejo de vingança, mas também, um aumento da evasão, dando indícios de que os clientes mantêm o rancor. Em segundo lugar, examinam o efeito de moderação de um relacionamento forte sobre o modo como os clientes mantêm esse rancor. Observam que os melhores clientes têm reações desfavoráveis mais longevas. A vingança de clientes de relacionamento forte diminui mais devagar, e sua evasão aumenta mais rapidamente do que a dos clientes de relacionamento fraco. Por fim, os autores exploram uma solução para atenuar esse efeito prejudicial, ou seja, a empresa oferece uma desculpa e uma compensação após a queixa online. Clientes de relacionamento forte são mais propensos à qualquer nível de tentativa de recuperação pós falha.

0 segundo artigo mais citado, Customer betrayal and retaliation: when your best customers became your worst enemies (Gregóire \& Fisher, 2008), propõe e testa um modelo baseado em justiça, que incorpora a traição percebida como meio para entender a retaliação do cliente e o efeito "amor torna-se ódio". Os resultados sugerem que a traição é uma força chave que leva os clientes a restaurar a equidade por todos os meios possíveis, incluindo retaliação.

Tabela 3 - Artigos mais citados.

\begin{tabular}{|c|c|c|c|c|c|}
\hline Artigos & Autores & Ano & Journal & $\begin{array}{l}\text { Fator de } \\
\text { impacto } \\
5 \text { anos }\end{array}$ & Citações \\
\hline $\begin{array}{l}\text { When Customer Love Turns into Last Hate: The Ef- } \\
\text { fects of Relationship Strength and Time on Cus- } \\
\text { tomer Revenge and Avoidance }\end{array}$ & $\begin{array}{l}\text { Gregóire, } \\
\text { Tripp \& Le- } \\
\text { goux }\end{array}$ & 2009 & $\begin{array}{l}\text { J. of Marke- } \\
\text { ting }\end{array}$ & 9.59 & 179 \\
\hline $\begin{array}{l}\text { Customer betrayal and retaliation: when your best } \\
\text { customers became your worst enemies }\end{array}$ & $\begin{array}{l}\text { Gregóire \& } \\
\text { Fisher }\end{array}$ & 2008 & $\begin{array}{l}\text { J. of the } \\
\text { Academy } \\
\text { of Market- } \\
\text { ing Science }\end{array}$ & 9.81 & 173 \\
\hline $\begin{array}{l}\text { The effects of perceived justice on recovery satis- } \\
\text { faction, trust, word-of-mouth, and revisit intention } \\
\text { in upscale hotels }\end{array}$ & $\begin{array}{l}\text { Kim, Kim \& } \\
\text { Kim }\end{array}$ & 2009 & $\begin{array}{l}\text { Tourism } \\
\text { Manage- } \\
\text { ment }\end{array}$ & 7,08 & 164 \\
\hline $\begin{array}{l}\text { The effect of compensation on repurchase inten- } \\
\text { tions in service recovery }\end{array}$ & $\begin{array}{l}\text { Grewal, } \\
\text { Roggeveen }\end{array}$ & 2008 & $\begin{array}{l}\text { J. of Retai- } \\
\text { ling }\end{array}$ & 5.53 & 83 \\
\hline
\end{tabular}


A comprehensive model of customer Direct and indirect revenge: understanding the effects of perceived greed and customer power

Customer rage episodes: emotions, expressions and behaviors

Why service recovery fails tension among customer, employee, and process perspectives

Perceived controllability and service expectations: influences on customer reactions following services failure

Anger, frustation, and helplessness after service failure: coping strategies and effective informational support

Customer complaining: the role of tie strength and information control

\begin{tabular}{|c|c|c|c|c|}
\hline \& Tsiros & & & & \\
\hline $\begin{array}{l}\text { Gregóire, } \\
\text { Laufer \& } \\
\text { Tripp }\end{array}$ & 2010 & $\begin{array}{c}\text { J. of the } \\
\text { Academy } \\
\text { of Market- } \\
\text { ing Science }\end{array}$ & 9.81 & 79 \\
\hline $\begin{array}{l}\text { McColl- } \\
\text { Kennedy, } \\
\text { Patterson, } \\
\text { Smith \& } \\
\text { Brady }\end{array}$ & 2009 & $\begin{array}{l}\text { J. of Retai- } \\
\text { ling }\end{array}$ & 5.53 & 79 \\
\hline $\begin{array}{l}\text { Michel, Bo- } \\
\text { wen \& } \\
\text { Johnston }\end{array}$ & 2009 & $\begin{array}{c}\text { J. of Service } \\
\text { Manage- } \\
\text { ment }\end{array}$ & 5.407 & 78 \\
\hline $\begin{array}{l}\text { Choi \& } \\
\text { Mattila }\end{array}$ & 2008 & $\begin{array}{c}\text { J. of Busi- } \\
\text { ness Rese- } \\
\text { arch }\end{array}$ & 3.689 & 73 \\
\hline Gelbrich & 2010 & $\begin{array}{l}\text { J. of the } \\
\text { Academy } \\
\text { of Market- } \\
\text { ing Science }\end{array}$ & 9.81 & 72 \\
\hline $\begin{array}{c}\text { Mittal, Hu- } \\
\text { ppertz \& } \\
\text { Khare }\end{array}$ & 2008 & $\begin{array}{l}\text { J. of Retai- } \\
\text { ling }\end{array}$ & 5.53 & 64 \\
\hline
\end{tabular}

Fonte: Elaborado pelos autores (2018).

Grégoire Yany emerge como o principal autor sobre falhas de serviços no período. Ele ocupa o posto de autor mais prolífico no período com sete publicações, e possui o primeiro e o segundo artigos mais citados nesta amostra. No cenário brasileiro, Celso Augusto de Matos, doutor em Administração pela Universidade Federal do Rio Grande do Sul, aparece na sexta posição entre os autores mais expoentes, com 4 artigos publicados entre 2009 e 2013. A relevância desses autores é evidenciada quando se observa que a amostra é composta por 436 autores.

Por meio da análise de citação, pode-se observar ainda, que embora exista um número considerável de artigos tratando sobre falha de serviços, o tema permanece atual, pulsante, e que novos estudos são necessários para dar resposta às novas faces da complexa relação empresa vs consumidor.

\subsection{Análise de Cocitação}

A análise de cocitação mede a ocorrência em que um par de referências é citado conjuntamente, de modo que, quanto maior a cocitação, maior é a semelhança entre as referências (Vogel, \& Güttel, 2013). Pode ainda identificar os principais pilares de um tema (Zupic, \& Čater, 2015), criando assim a trajetória percorrida pelo tema.

Por meio do software Bibexcel (Pilkington, 2006), criou-se uma matriz de cocitação. Em seguida, realizou-se uma análise fatorial exploratória, processada com o software SPSS, método usual para bibliometrias (Vogel, \& Güttel, 2013). Utilizou-se a rotação Varimax, de modo a reduzir e agrupar as variáveis em fatores com grande relação entre si (Hair, Black, Babin, \& Anderson, 2014). Foram descartadas cargas fatoriais abaixo de 0,50 , bem como artigos com cargas fatoriais presentes em mais de dois fatores. A análise resultou em três fatores, conforme a Tabela 4, representando as abordagens teóricas usadas na amostra. 0 valor de KMO da análise foi de 0,882. Após a leitura dos artigos, de modo a validar e compreender a maneira como se conectam, os fatores foram nomeados da seguinte maneira: (1) Qualidade do encontro; (2) Percepção de sentimentos; e (3) Encontros de Recuperação. Os três fatores identificados explicam $76,48 \%$ da variância.

O Fator 1, com nove artigos, representa 30,529\% da variância total explicada. Dos nove artigos, oito foram publicados na década de 1990, e representam um resumo da base seminal desta análise. Esses artigos focam, principalmente, no efeito que a qualidade do encontro produz sobre a satisfação do consumidor. A complexidade e a intangibilidade observadas nas relações de serviço são destacadas, e a percepção e inferência que o consumidor atribui a esse encontro norteiam a avaliação que será feita. 0 artigo Critical service encounters: The employee's viewpoint (Bitner, Booms, \& Mohr, 1994) possui maior 
carga fatorial (.902) no fator 1. Os autores propõem que a satisfação do consumidor é influenciada pela qualidade da interação no momento do encontro. É interessante observar que os autores salientam um contraponto ao pensamento vigente na década de 1990, em que o "cliente é rei", e assim, estimulam o aprofundamento na compreensão da relação empresa vs cliente.

\section{Tabela 4 - Análise Fatorial Exploratória - Cocitação.}

\begin{tabular}{|c|c|c|}
\hline Qualidade do encontro & Percepções e sentimentos & Encontros de recuperação \\
\hline $\begin{array}{l}\text { Bitner, Booms, \& Mohr (1994) .902 } \\
\text { Bitner, Booms, \& }\end{array}$ & $\begin{array}{l}\text { Folkes, Koletsky \& } \\
\text { Graham (1987) } \\
\text { Bougie, Pieters \& }\end{array}$ & $\begin{array}{l}\text { Smith, Bolton \& } \\
\text { Wagner (1999) }\end{array}$ \\
\hline Tetreault (1990) & Zeelenberg (2003) .823 & Smith \& Bolton (2002) \\
\hline Bitner (1990) & Bonifield \& Cole (2007) .822 & Smith \& Bolton (1998) \\
\hline $\begin{array}{l}\text { Grongroos (1988) } \\
\text { Spreng, Harrell, \& } \\
\text { Mackoy (1995) } \\
\text { Kelley (1995) }\end{array}$ & $\begin{array}{lc}\text { Gregoire, Tripp } & \\
\text { \& Legoux (2009) } & .795 \\
\text { Gregóire \& } & \\
\text { Fisher (2008) } & .750 \\
\text { Tax \& Brown (1998) } & .577\end{array}$ & \\
\hline $\begin{array}{l}\text { Zeithaml, Berry, \& } \\
\text { Parasuraman (1996) } \\
\text { Hart, Heskett \& } \\
\text { Sasser (1990) } \\
\text { Maxham III \& } \\
\text { Netemeyer (2002) }\end{array}$ & & \\
\hline Variância Explicada 30,529\% & Variância explicada $28,133 \%$ & Variância Explicada 19,98\% \\
\hline
\end{tabular}

Fonte: Elaborado pelos autores (2018).

O Fator 2 explica 28,133\% da variância, e é composto por seis artigos. 0 ponto de convergência para os artigos agrupados no fator 2 é observação dos sentimentos negativos gerados a partir de uma falha de serviços. Aspectos como raiva e vingança são abordados. 0 artigo A field study of causal inferences and consumer reaction: the view from the airport (Folkes, Koletsky, \& Graham, 1987) possui maior carga fatorial (.827) e é o artigo mais antigo do fator 2. Os autores entrevistam 97 passageiros de voos atrasados objetivando examinar as respostas afetivas e comportamentais desses consumidores diante da falha de serviços. Demonstram, com este estudo, que as inferências sobre o porquê produtos falham influenciam o grau de raiva que os consumidores sentem, assim como, também influenciam o desejo de recomprar e reclamar do problema. Gregóire, Tripp e Legoux (2009) trazem uma observação longitudinal e avaliam os efeitos do tempo e da força do relacionamento sobre a evolução do desejo de vingança e evasão dos clientes.

O Fator 3 possui 3 artigos, todos tendo por primeira autora Amy K. Smith, e explica 19,98\% da variância. Esses artigos indicam que as respostas emocionais dos clientes às falhas do serviço influenciarão os esforços de recuperação e julgamentos de satisfação. A model of customer satisfaction with service encounters involving failure and recovery (Smith, Bolton, \& Wagner, 1999) apresenta a maior carga fatorial (.906) no terceiro fator. A temática central do artigo é a recuperação da satisfação do consumidor após uma falha de serviços. Como principal resultado do estudo, observa-se que consumidores esperam que as reparações devem ser proporcionais a magnitude da falha.

Por meio da análise de cocitação pode-se observar os pilares da estrutura conceitual desta amostra. 0 fator 1 agrupou, em sua maioria, os artigos mais antigos da amostra, e tem por foco central a ocorrência da falha de serviços, avaliando os aspectos negativos nas relações com os consumidores. 0 fator 2 reúne artigos que oferecem uma atenção especial aos sentimentos gerados pela falha de serviços, sem o entendimento dos quais, aumenta o grau de dificuldade na recuperação dos clientes. Por fim, o fator 3 agrupa artigos que tratam da recuperação pós falha, indicando que a reparação é o meio mais efetivo para tal.

A Figura 4 representa a rede de cocitação com os trabalhos mais citados, e foi elaborada com o software UciNet. As figuras indicam os artigos, e as linhas as relações de cocitação. Os artigos com maior grau de centralidade são mais influentes. Dois artigos se destacam, e os demais artigos orbitam a seu redor. Tax e Brown (1998) oferecem um guia propondo diretrizes para a recuperação dos clientes. Hart, Heskett e Sasser (1990) avaliam a rapidez na identificação e resposta oferecida pela empresa diante de 
uma falha de serviços.

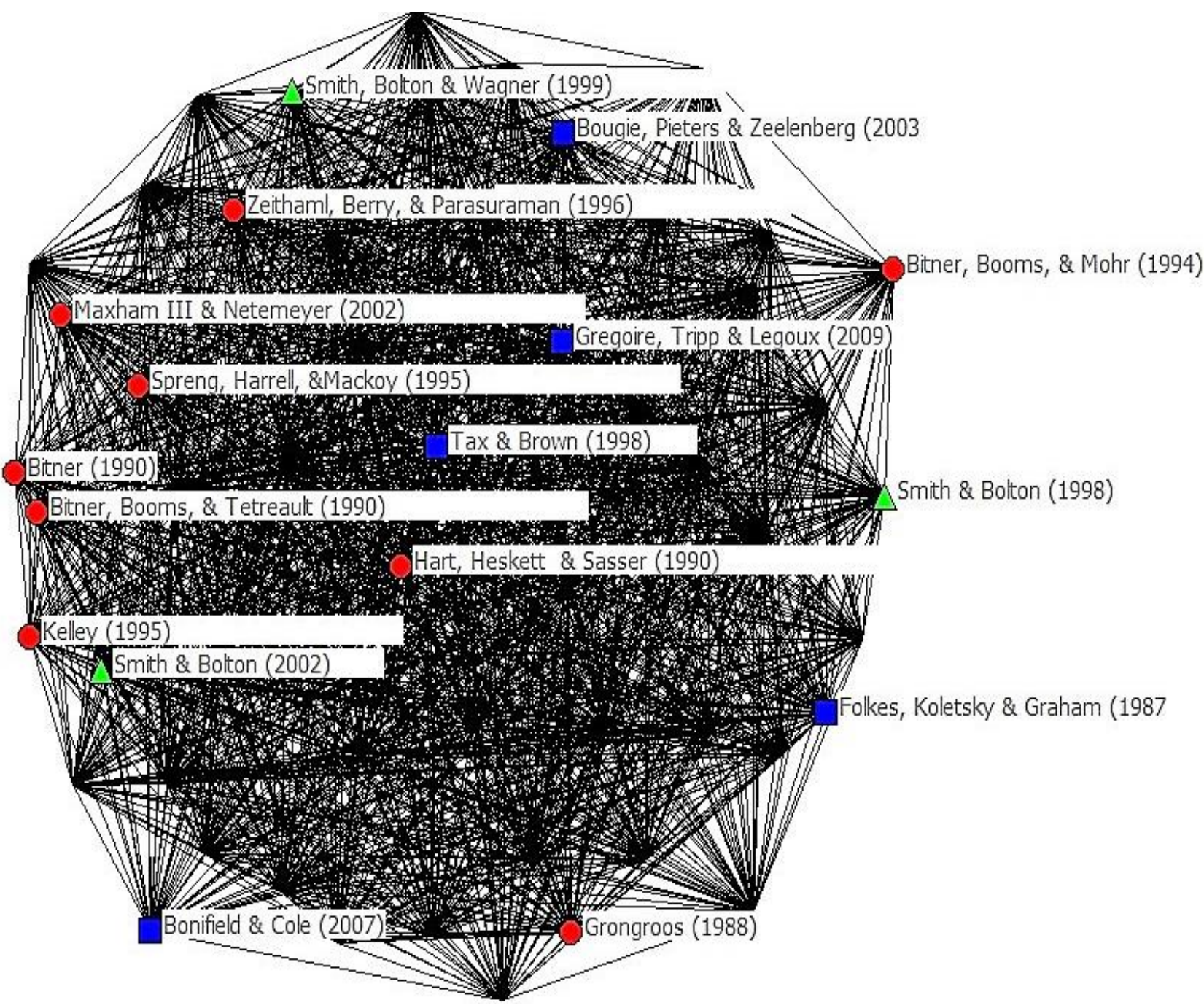

\section{Qualidade do encontro}

Figura 4: Rede de Cocitação

Fonte: Elaborado pelos autores

\subsection{Análise de Pareamento}

O pareamento bibliográfico mede o uso de uma mesma referência por dois ou mais artigos, e permite um entendimento da tendência do tema, em função do que foi publicado. 0 método de análise utilizado assemelha-se à análise de cocitação. A partir dos 199 artigos da amostra, criou-se uma matriz de pareamento com o software Bibexcel (Pilkington, 2006). Após, foi realizada a análise fatorial exploratória com o software SPSS (versão 18), com extração de fatores pelo método de rotação Varimax. Foram descartadas cargas fatoriais abaixo de 0,50 , bem como artigos com cargas fatoriais presentes em mais de dois fatores. A análise fatorial resultou no agrupamento dos artigos em oito fatores. 0 valor de KMO da análise foi de .837, e 61,88\% foi o percentual de variância explicada. A Tabela 5 apresenta a análise fatorial exploratória.

0 primeiro fator possui 18 artigos e apresenta o maior percentual sobre a variância explicada, 19,67\%. A temática central do fator, tange a multiplicidade de respostas dos consumidores, diante de uma recuperação de falha de serviços. A individualidade observada nas respostas dos consumidores no processo de recuperação, mantem viva a necessidade de continuidade dos estudos sobre o tema. Os autores agrupados neste fator testam aspectos distintos, e oferecem explicações para conflitos pontuais. 
Dentre a variedade de abordagens, destacam-se: percepções de justiça (Kim, Kim \& Kim, 2009; Nikbin, Armesh, Heydari \& Jalalkamali, 2011; Aguilar-Rojas, Fandos-Herrera, Flavian-Blanco, 2015; Gohary, Hamzelu \& Pourazizi, 2016; Jung \& Seock, 2017), compensação reparatória (Lii \& Lee, 2012; BambauerSachse \& Rabeson, 2015; Gelbrich. Gathke \& Grégoire, 2016; e Sharifi \& Aghazadeh, 2016) e relacionamento (Kruger, Mostert \& Beer, 2015; Moliner-Velázquez, Ruiz-Molina, \& Fayos-Gardó, 2015; e CambraFierro, Melero-Polo, \& Sese, 2016). 0 primeiro fator foi nomeado recuperação de clientes.

Tabela 5 - Análise Fatorial Exploratória - Pareamento.

\begin{tabular}{|c|c|c|c|c|c|c|c|c|}
\hline Artigo & $\begin{array}{c}\text { Fator } \\
1\end{array}$ & $\begin{array}{l}\text { Fator } \\
2\end{array}$ & $\begin{array}{c}\text { Fator } \\
3\end{array}$ & $\begin{array}{c}\text { Fator } \\
4\end{array}$ & $\begin{array}{c}\text { Fator } \\
\mathbf{5}\end{array}$ & $\begin{array}{c}\text { Fator } \\
6\end{array}$ & $\begin{array}{c}\text { Fator } \\
7\end{array}$ & $\begin{array}{c}\text { Fator } \\
8\end{array}$ \\
\hline Jung \& Seock (2017) & .778 & & & & & & & \\
\hline Chueng \& To (2016) & .776 & & & & & & & \\
\hline Nikbin, Armesh, Heydari \& Jalalkamali (2011) & .775 & & & & & & & \\
\hline Lii \& Lee (2012) & .700 & & & & & & & \\
\hline $\begin{array}{c}\text { Bougoure, Russell-Bennett, } \\
\text { Fazal-E-Hasan \& Mortimer (2016) }\end{array}$ & .697 & & & & & & & \\
\hline Gohary, Hamzelu \& Pourazizi (2016) & .692 & & & & & & & \\
\hline $\mathrm{Xu}$, Tronvoll \& Edvardsson (2014) & .688 & & & & & & & \\
\hline Tarofder, Nikhashemi, \& Azam (2016) & .685 & & & & & & & \\
\hline Sharifi \& Aghazadeh (2016) & .674 & & & & & & & \\
\hline Kruger, Mostert \& Beer (2015) & .631 & & & & & & & \\
\hline Cambra-Fierro, Melero-Polo, \& Sese (2016) & .630 & & & & & & & \\
\hline $\begin{array}{l}\text { Moliner-Velázquez, Ruiz-Molina, \& } \\
\text { Fayos-Gardó (2015) }\end{array}$ & .616 & & & & & & & \\
\hline Tektas (2017) & .616 & & & & & & & \\
\hline Kim, Kim \& Kim (2009) & .612 & & & & & & & \\
\hline Bambauer-Sachse \& Rabeson (2015) & .604 & & & & & & & \\
\hline $\begin{array}{l}\text { Aguilar-Rojas, Fandos-Herrera, Flavian-Blanco } \\
\qquad(2015)\end{array}$ & .602 & & & & & & & \\
\hline Silva, Broilo, Spartel, \& Basso (2017) & .592 & & & & & & & \\
\hline Gelbrich. Gathke \& Grégoire (2016) & .567 & & & & & & & \\
\hline Schoefer \& Diamantopoulos (2008) & & .758 & & & & & & \\
\hline Wen \& Geng-ging Chi (2013) & & .750 & & & & & & \\
\hline Riscinto Kozub, Anthony O'Neill \& Palmer & & .702 & & & & & & \\
\hline Gabbott, Tsarenko \& Mok (2011) & & .664 & & & & & & \\
\hline Sanchez-Garcia \& Curras-Perez (2011) & & .653 & & & & & & \\
\hline Jean Harrison-Walker (2012) & & .647 & & & & & & \\
\hline Ozgen \& Duman Kurt (2012) & & .637 & & & & & & \\
\hline Keeffe, Russel-Bennett \& Tombs (2008) & & .635 & & & & & & \\
\hline Michel, Bowen \& Johnston (2009) & & & .726 & & & & & \\
\hline Tsarenko \& Rooslani Tojib (2011) & & & .723 & & & & & \\
\hline Namkung \& Jang (2010) & & & .624 & & & & & \\
\hline Soares, Zhang, Proença \& Kandampully (2017) & & & .584 & & & & & \\
\hline Morrisson \& Huppertz (2010) & & & .549 & & & & & \\
\hline Kim \& Jang (2016) & & & & .731 & & & & \\
\hline Chen, Gerstner \& Yang (2012) & & & & .717 & & & & \\
\hline Sivakumar, Li \& Dong (2014) & & & & .643 & & & & \\
\hline Chuang, Cheng, Chang \& Yang (2012) & & & & .524 & & & & \\
\hline $\begin{array}{c}\text { van Vaerenbergh, Orsingher, Vermeir \& Larivi- } \\
\text { ere (2014) }\end{array}$ & & & & & .830 & & & \\
\hline $\begin{array}{c}\text { Varela-Neira, Vázquez-Casielles, \& Iglesias, } \\
\text { (2014 }\end{array}$ & & & & & .704 & & & \\
\hline Choi \& Matila (2008) & & & & & .630 & & & \\
\hline La \& Choi (2012) & & & & & & .722 & & \\
\hline Fatma, Khan \& Rahman (2016) & & & & & & .607 & & \\
\hline Choi \& La (2013) & & & & & & .528 & & \\
\hline Grégoire, Tripp \& Legoux (2009) & & & & & & & .845 & \\
\hline Joireman, Grégoire, Devezer \& Tripp (2013) & & & & & & & .772 & \\
\hline Lopes \& Silva (2015) & & & & & & & & .795 \\
\hline VARIÂNCIA EXPLICADA (\%) & 19,67 & 10,58 & 6,93 & 6,67 & 5,71 & 4,98 & 4,30 & 3,00 \\
\hline
\end{tabular}

Fonte: elaborada pelos autores (2018).

Resposta emocional é a denominação do segundo fator. Composto por nove artigos, explica 10,58\% da variância. O papel desempenhado pelas emoções é tema central dos artigos deste fator. Os 
autores avaliam o papel preditor das emoções sobre a recuperação pós falha de serviços. Schoefer e Diamantopoulos (2008) propõem uma escala para a mensuração das emoções vivenciadas durante o processo de recuperação do cliente. Gabbott, Tsarenko e Mok (2011) observam a inteligência emocional na resposta dos clientes frente a falha e recuperação.

0 terceiro fator explica 6,93\% da variância, e é composto por cinco artigos. Nomeado tensões relacionais, agrupa artigos que analisam as tensões decorrentes de uma falha de serviço, e que permeiam o processo de recuperação. Esses artigos trazem prismas distintos, que propiciam uma maior amplitude de análise. Michel, Bowen e Johnston (2009) identificam as tensões específicas entre cliente, processo e recuperação por funcionários. Tsarenko e Rooslani Tojib (2011) exploram o conceito de perdão diante de uma falha. Namkung e Jang (2010) abordam os diversos estágios de um restaurante frente a falha de serviços, identificando pontos críticos, e favorecendo a recuperação e manutenção dos clientes. Soares, Zhang, Proença e Kandampully (2017) estudam como os grupos de gerações (geração silenciosa, Baby Boomers, geração X e geração Y) respondem às falhas de serviço e a recuperação pós falha. Morrisson e Huppertz (2010) estudam clientes do programa de fidelidade.

Contabilidade mental é o nome do quarto fator. Possui quatro artigos, e explica 6,67\% da variância. $\mathrm{O}$ foco central deste fator é análise da memória que o cliente mantém sobre a falha de serviços, e o consequente esforço dispendido na recuperação. 0 artigo Factors affecting memorability of service failures: a longitudinal analysis (Kim \& Jang, 2016) propõe que as recuperações de serviço diminuem a memorização da falha ocorrida, e por consequência, diminuem os aspectos negativos para o cliente.

0 quinto fator é composto por três artigos e representa 5,71\% da variância explicada. Nomeado capacidade de controle, os artigos convergem para uma análise sobre o valor de atribuição formado pelo consumidor. Van Vaerenbergh, Orsingher, Vermeir e Lariviere (2014) desenvolveram uma meta análise, e sugerem que, quando clientes atribuem que a falha poderia ser evitada, ocorre um impacto negativo sobre o nível de satisfação.

Lealdade é o nome do sexto fator. Composto por três artigos, explica 4,98\% da variância. La e Choi (2012) sugerem que a reconstrução da lealdade após uma falha de serviços depende da recuperação da confiança, e que a principal determinante para a recuperação da confiança é a afeição do cliente. Fatma, Khan e Rahman (2016) e Choi e La (2013) analisam a conexão entre responsabilidade social corporativa e lealdade.

0 sétimo fator, nomeado resposta à recuperação, é formado por dois artigos, e explica $4,30 \%$ da variância. Os autores analisam o modo como os clientes respondem a uma recuperação após uma falha de serviços. No artigo de 2009, Grégoire, Tripp e Legoux observam o desejo de vingança e evasão dos clientes em função do tempo e do relacionamento. No artigo de 2013, Joireman, Grégoire, Devezer e Tripp estudam o impacto causado pela inferência do motivo pela falha de serviços.

0 oitavo fator, nomeado justiça percebida, é composto por um artigo de 2015 , e explica $3 \%$ da variância. Lopes e Silva (2015) observam o papel da justiça percebida como antecedente da lealdade. Os autores demonstram a significância da justiça distributiva e da justiça interacional sobre satisfação, qualidade percebida e confiança do consumidor. Sugerem também, que o processo de comportamento dos reclamantes como oportunidade para fortalecer a relação com os clientes.

Por meio da análise de pareamento, foi possível observar as atuais preocupações com o tema falha de serviços. A amostra que compõe essa análise é formada por artigos publicados entre 2008 e 2017. A temática central destes artigos é a recuperação pós falha de serviços. Se por um lado a literatura dá conta em responder os aspectos que tangem a falha de serviços (análise de cocitação), ainda existem lacunas a serem preenchidas quanto à recuperação pós falha. 0 crescente volume de publicações sobre o tema a partir de 2012 corrobora essa necessidade. 0 principal fator de negativa para o esgotamento do tema recuperação pós falha, é o fato de consumidores responderem de modo diferente, frente aos diferentes tipos de recuperação. Alguns pontos já estão consolidados, tais como a reparação tangível pela falha, mas, mesmo sob esse ponto factual, decorrem análises distintas por conta da percepção sobre a equivalência entre reparação e falha, bem como percepção de cada ator envolvido.

A Figura 5 representa a rede relacional da análise de pareamento bibliográfico, e foi construída com o uso do software UciNet. A rede relacional referente a análise de pareamento bibliográfico expõe o agrupamento da maioria dos artigos do fator 1 na parte esquerda do mapa, assim como a maior parte dos artigos publicados em 2015, 2016 e 2017, indicando uma concentração na busca pelo entendimento das percepções dos consumidores, e, por consequência, uma maior abrangência nas vertentes utilizadas. 
Assim, pode-se sugerir que a busca por novos prismas para a avaliação das falhas de serviço é uma tendência para este campo de pesquisa.
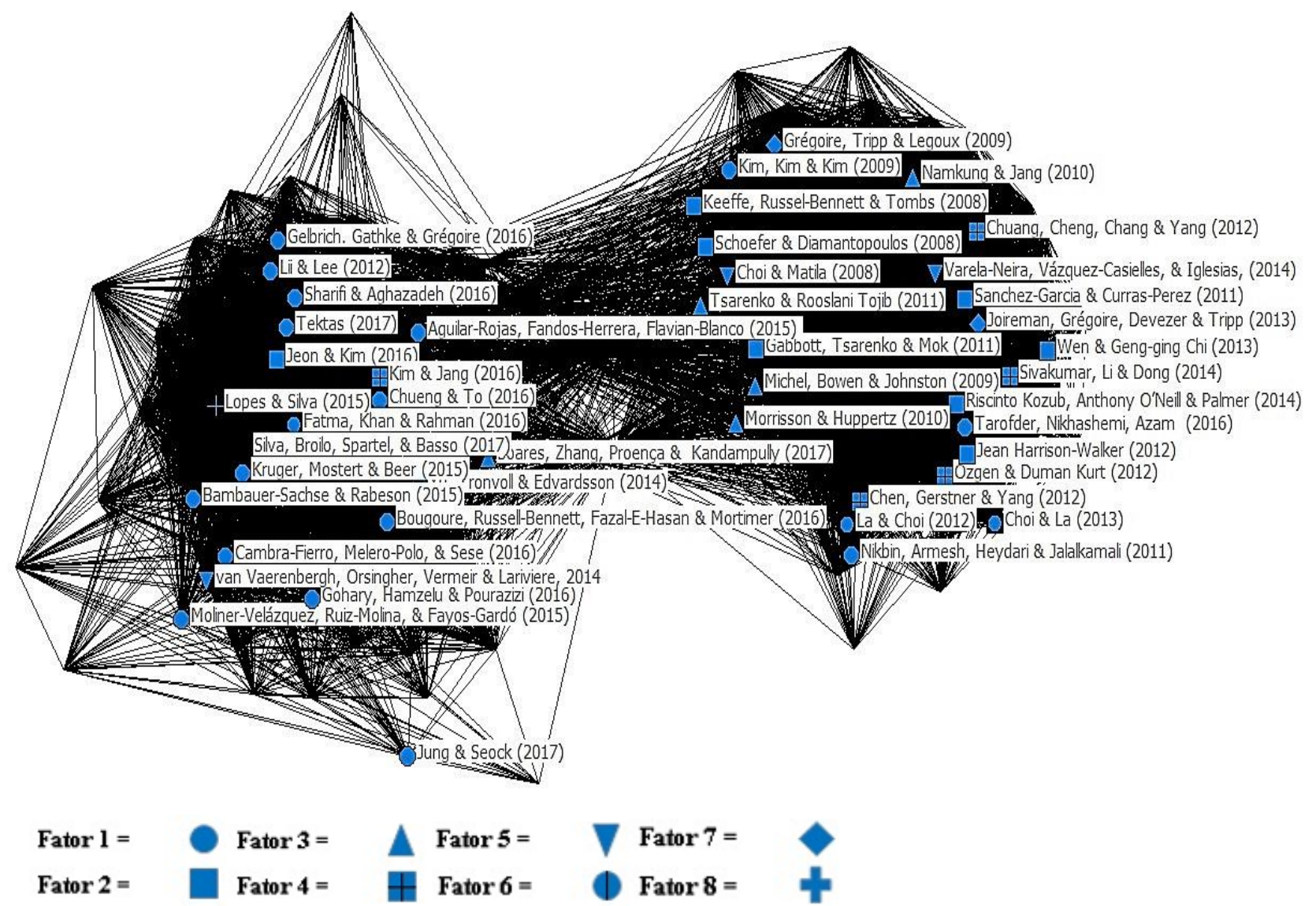

Figura 5: Rede de Pareamento Bibliográfico

Fonte: Elaborado pelos autores (2018).

\section{Conclusão}

O objetivo deste estudo foi identificar as pesquisas mais significativas sobre falha de serviços no período de 2008 a 2017. Realizou-se uma bibliometria, em uma amostra de 199 artigos publicados no período, coletados no portal Web of Science. Três análises foram realizadas: citação, que indicou o autor Gregóire Yane como o autor de maior destaque no período, pois além de ocupar o posto de autor mais prolífico, escreveu o artigo mais citado; cocitação, que indicou três principais abordagens teóricas para a amostra, (1) qualidade do encontro, (2) percepção de sentimentos, e (3) encontros de recuperação, construindo um entendimento robusto sobre as implicações geradas pelas falhas no relacionamento com o cliente, bem como procedimentos para a recuperação desses clientes; e pareamento bibliográfico, que indicou a efervescência existente sobre o tema e o uso de uma maior amplitude nas vertentes utilizadas nas abordagens, refletindo o aumento na complexidade observada na relação empresa e cliente.

Este estudo apresenta um levantamento dos artigos que reforçam os impactos negativos que a falha de serviços exerce sobre o consumidor, resultando em sentimentos ruins, tais como, raiva, insatisfação e retaliação, bem como os esforços realizados na recuperação desses clientes. Com a análise de cocitação pode-se destacar os principais artigos que compõem a trajetória de estudos sobre o tema. Com a análise de pareamento pode-se observar que o tema, embora bastante estudado, ainda precisa ser abordado por novos prismas. À medida que outros componentes surgem no cenário, novos estudos serão necessários para dar respostas as essas demandas. 0 campo de pesquisas sobre o tema falha de serviços permanece vivo, indicando sua relevância. Em termos gerenciais, este trabalho oferece aos gestores um guia dos principais artigos sobre falha de serviços, bem como um alerta para que considerem esforços contínuos na reparação de inevitáveis falhas de serviço.

Como limitações e sugestões para pesquisas futuras, esta pesquisa restringiu a seleção dos artigos 
apenas para as categorias Business e Management da base de dados da Web of Science. Pesquisas futuras podem expandir as categorias selecionadas. Para a análise de citação, não foi considerado o cenário em que a citação foi utilizada. Assim, um artigo pode ter sido citado para embasar um argumento, mas também pode ter sido citado de modo contrário. Embora a análise de cocitação permita, de modo positivo, a identificação e agrupamento de autores que se compatibilizem em citar as mesmas referências, também oferece uma limitação ao classificar apenas uma parte do rol de artigos citados, trazendo assim subjetividade na análise.

\section{APOIO}

"O presente trabalho foi realizado com apoio da Coordenação de Aperfeiçoamento de Pessoal de Nível Superior -Brasil (CAPES) - Código de Financiamento 001"

\section{NOTA}

Os autores agradecem aos avaliadores anônimos da TPA pelas sugestões de melhoria realizas nas fases inicias de avaliação deste trabalho.

\section{Referências}

Agarwal, R, Mehrotra, A., \& Barger, V.A. (2016). Personality traits and repatronage intentions after service failure. Journal of Consumer Satisfaction, Dissatisfaction \& Complaining Behavior, 29(1), 31-51.

Andrés, A. (2009). Measuring academic research: How to undertake a bibliometric study. Elsevier.

Araújo, C. A. A. (2006). Bibliometria: evolução histórica e questões atuais. Em Questão, 12(1).

Bies, R.J., \& Shapiro, D.L. (1987), International fairness judgments: the influence of causal accounts, Social Justice Research, 1(2), 199-218.

Casado-Diaz, A.B., \& Nicolau-Gonzálbez, J.L. (2009). Explaining consumer complaining behaviour in double deviation scenarios: the banking services. The Service Industries Journal, 29(12), 1659-1668.

Choi, B., \& Choi, B. J. (2014). The effects of perceived service recovery justice on customer affection, loyalty, and word-of-mouth. European Journal of Marketing, 48(1/2), 108-131.

Egghe, L., \& Rousseau, R. (2002). Co-citation, bibliographic coupling and a characterization of lattice citation networks. Scientometrics, 55(3), 349-361.

Grégoire, Y \& Fisher, R.J. (2008). Customer betrayal and retaliation: when your best customers become your worst enemies. Journal of the Academy of Marketing Science. 36(2), 247- 261.

Grégoire, Y., Tripp, T.M. \& Legoux, R. (2009). When customer love turns into lasting hate: the effects of relationship strength and time on customer revenge and avoidance. Journal of Marketing, 73(11), 1832.

Hair, J. F., Black, W. C., Babin, B. J., \& Anderson, R. E. (2014). Exploratory factor analysis. Multivariate data analysis, 7th Pearson new international ed. Harlow: Pearson.

Hennig-Thurau, T., Gwinner, K. P., \& Gremler, D. D. (2002). Understanding Relationship Marketing Outcomes: An Integration of Relational Benefits and Relationship Quality. Journal of Service Research, $4(3), 230-247$.

Huang, S.W., Hung, Y.W., Fu, T.W., Hsu, J.S.C., \& Chiu, M.C. (2015). Understanding the impact of service failure and recovery justice on consumer's satisfaction and repurchase intention. The 19th Pacific Asia Conference on Information Systems (PACIS). Singapore.

Hung, S. W., Cheng, M. J., \& Chiu, P. C. (2019). Do antecedents of trust and satisfaction promote consumer loyalty in physical and virtual stores? a multi-channel view. Service Business, 1-23.

Jung, N.Y., Seock, Y.K. (2017). Effect of service recovery on customers' perceived justice, satisfaction, and word-of-mouth intentions on online shopping websites. Journal of Retailing and Consumer Services. 37(1), 23-30.

Katz, J. S., \& Hicks, D. (1997). How much is a collaboration worth? A calibrated bibliometric model. Scientometrics, 40(3), 541-554.

Keaveney, S.M. (1995). Customer switching behavior in service industries: an exploratory. Journal of Marketing, 59(4), 71-82.

Leydesdorff, L. (2009). How are new citation-based journal indicators adding to the bibliometric toolbox? Journal of the American Society for Information Science and Technology, 60(7), 1327-1336.

Lim, E.A.C., Lee, Y.H., \& Foo, M.D. (2017). Frontline employees' nonverbal cues in service encounters: a double-edged sword. Journal of the Academy of Marketing Science, 45(5), 657-676. 
Lin, T., \& Cheng, Y. (2010). Exploring the knowledge network of strategic alliance research: A co-citation analysis. International Journal of Electronic Business Management, 8(2), 152- 160.

Lorenz, P. M., Johnson, C. M., \& Barakat, L. L. (2017). An exploration of frequent service failures in the Brazilian airline industry. Latin American Business Review, 18(1), 19-45.

Lovelock, C. H., \& Wirtz, J. (2007). "Services marketing: people, technology, strategy", (6 ed.), PrenticeHall

Mattila, A.S., \& Patterson, P.G. (2004), The impact of culture on consumers, perceptions of service recovery efforts", Journal of Retailing, 80(3), 196-206.

McColl-Kennedy, J.R., \& Sparks, B. A. (2003). Application of service failures and service recovery. Journal of Business Research, 5, 251-266.

Nikbin, D., Marimuthu, M., Hyun, S. S., \& Ismail., I. (2015). Relationships of perceived justice to service recovery, service failure attributions, recovery satisfaction, and loyalty in the context of airline travelers. Asia Pacific Journal of Tourism Research, 20(3), 239-262.

Nwokorie, E. C. (2016). Service recovery strategies and customer loyalty in selected hotels in Lagos state, Nigeria. Net Journal of Business Management, 4(1), 1-8.

Oliver, R.L. (1980). A cognitive model of the antecedents and consequences of satisfaction decisions. Journal of Marketing Research. 17(4). 460 - 469.

Oliver, R.L., \& Swan, J.E. (1989). Consumer perceptions of interpersonal equity and satisfaction in transactions: a field survey approach. Journal of Marketing, 53(2), 21-35.

Orsingher, C., Valentini, S., de Angelis, M. (2010), A meta-analysis of satisfaction with complaint handling in services. Journal of the Academy of Marketing Science, 38(2), 169-186.

Parasuraman, A, Zeithmal, V. A., \& Berry, L. L. (1990). Delivering service quality: balancing customer perceptions and expectations. New York: The Free Press.

Park, E. (2019). The role of satisfaction on customer reuse to airline services: An application of Big Data approaches. Journal of Retailing and Consumer Services, 47, 370-374.

Ramos-Rodríguez, A.-R., \& Ruíz-Navarro, J. (2004). Changes in the intellectual structure of strategic management research: a bibliometric study of the Strategic Management Journal, 1980-2000. Strategic Management Journal, 25(10), 981-1004.

Rao, S., Griffis, S.E., \& Goldsby, T.J. (2011). Failure to deliver? Linking online order fulfillment glitches with future purchase behavior. Journal of Operations Management. 29(8), 692-703.

Rasoulian, S., Grégoire, Y., Legoux, R., \& Sénécal, S. (2017). Service crisis recovery and firm performance: insights from information breach announcements. Journal of the Academy of Marketing Science, 45(6), 789-806.

Ro, H. (2014) Complaint, patience, and neglect: responses to a dissatisfying service experience. Service Business, 8(2), 197-216.

Silva, F.Q., Santos, E.B.A, Brandão, M.M., \& Vils, L. (2016) Estudo bibliométrico: orientações sobre sua aplicação. Revista Brasileira de Marketing - ReMark, 15(2), 246-262.

Silva, R.G.S., Broilo, P.L., \& Espartel, L.B. (2017). Altruistic punishment: a consumer response to service failure. Journal of Marketing Theory and Practice, 25(4), 421-435.

Tarofder A.K., Nikhashemi, S.R., Azam, S.M.F., \& Haque, P.S.A. (2016). "The mediating influence of service failure explanation on customer repurchase intention through customers satisfaction", International Journal of Quality and Service Sciences, 8(4), 516-535.

Vogel, R., \& Güttel, W. H. (2013). The dynamic capability view in strategic management: a bibliometric review. International Journal of Management Reviews, 15(4), 426-446.

Ward, J. C., \& Ostrom, A.L. (2006). "Complaining to the masses: the role of protest framing in customercreated complaint web sites," Journal of Consumer Research, 33(1), 220-30.

Zeithaml, V. and Bitner, M. (2003) Service marketing: integrating customer focus across the firm. New York: McGraw-Hill.

Zupic, I., \& Čater, T. (2015). Bibliometric methods in management and organization. Organizational Research Methods, 18(3),429-472. 